\title{
Development of Blood Glucose Control for Extremely Premature Infants
}

Aaron J. Le Compte ${ }^{1}$, J. Geoffrey Chase ${ }^{1}$, Adrienne Lynn², Chris E. Hann ${ }^{1}$, Geoffrey M. Shaw $^{3}$, Jessica Lin ${ }^{1}$

1

PhD, Department of Mechanical Engineering, University of Canterbury, New Zealand 2

MBChB, FRACP, Neonatal Department, Christchurch Women's Hospital, New Zealand 3

MbChB, FJFICM, Department of Intensive Care, Christchurch Hospital, New Zealand

Work performed at:

- Neonatal Department, Christchurch Hospital

- Department of Mechanical Engineering, University of Canterbury

- Christchurch School of Medicine and Health Sciences, University of Otago

Address for Correspondence:

Prof J. Geoffrey Chase,

Department of Mechanical Engineering

University of Canterbury,

Private Bag 4800

Christchurch

New Zealand

Email: geoff.chase@canterbury.ac.nz

Phone: +64-3-364-2987 xtn 7224

Keywords: Glucose, simulation, insulin, control, real-time, neonate, user interface. 


\section{Abstract:}

Extremely premature neonates often experience hyperglycaemia, which has been linked to increased mortality and worsened outcomes. Insulin therapy can assist in controlling blood glucose levels and promoting needed growth. This study presents the development of a model-based stochastic targeted controller designed to adapt insulin infusion rates to match the unique and changing metabolic state and control parameters of the neonate. Long-term usage of targeted BG control requires successfully forecasting variations in neonatal metabolic state, accounting for differences in clinical practices between units, and demonstrating robustness to errors that can occur in everyday clinical usage. Simulation studies were used to evaluate controller ability to target several common BG ranges and evaluate controller sensitivity to missed BG measurements and delays in control interventions on a virtual patient cohort of 25 infants developed from retrospective data. Initial clinical pilot trials indicated model performance matched expected performance from simulations. Stochastic targeted glucose control developed using validated patient-specific virtual trials can yield effective protocols for this cohort. Long-term trials show fundamental success, however clinical interface design appears as a critical factor to ensuring good compliance and thus good control. 


\section{INTRODUCTION}

Metabolic homeostasis in the extremely premature infant is often compromised by immaturity of control systems. Up to 32\%-80\% of low birth weight infants experience hyperglycaemia with glucose levels exceeding thresholds of 6.9 to $13.9 \mathrm{mmol} / \mathrm{L}$ during the neonatal period [1-5]. The risk of hyperglycaemia is at least 18 times greater in infants with birth-weight less and 1,000 grams compared to infants weighing greater than 2,000 grams [6]. Hyperglycaemia has been linked to worsened outcomes. Associated morbidities include osmotic diuresis, electrolyte imbalance, intraventricular haemorrhage, sepsis, and increased ventilator dependence, retinopathy of prematurity, hospital length of stay and mortality [2-5, 7-9]. High rates of proteolysis are also common in low birth weight infants, reducing muscle mass and inhibiting growth [10].

The known mechanisms responsible for hyperglycaemia specific to extremely premature infants are related to their reduced ability to produce insulin [11]; defective beta-cell processing of pro-insulin (which is 10-16 times less active than insulin) to insulin [12]; an inability to suppress hepatic glucose production in response to glucose infusion [13]; and, finally, a decreased uptake of glucose secondary to a limited mass of insulin-sensitivity tissues (e.g.: muscle and adipose tissue) [14]. In addition to these factors, it has been shown that preterm infants can mount a hormonal response to stress similar to older critically ill patients [15]. Inhibiting the physiological response to reduce increased glycaemic levels are factors such as increased insulin resistance, absolute or relative insulin deficiency, and drug therapy [16-19].

Blood glucose control for the neonate poses several challenges that differ from the adult critical care case. Blood volumes in preterm infants are relatively small [6]. Thus, the number of blood glucose measurements must be optimised to a minimum useful number to conserve volume and restrict opening incubator doors, which may affect the neonate's hydration status [20]. Endogenous 
energy substrates are very low in preterm infants at birth [21]. Thus, these infants must be constantly fed to provide enough energy for basal requirements in addition to growth. In contrast, adults can tolerate periods of reduced caloric intake. Less saturation of the insulin-stimulated glucose uptake pathway has also been reported in neonates [22], unlike the saturation in adults [23]. Finally, also unlike the adult case, growth is a major goal of neonatal care. Thus, the anabolic effects of insulin are of relatively higher importance that in adults [10].

A small number of prospective trials have used insulin infusions to treat hyperglycaemia and/or promote growth [16, 24-32]. All reported insulin infusion trials have used either protocols that fixed insulin dosing to weight or other factors [25], or clinician judgment to determine insulin infusion rates. Positive outcomes of insulin infusion have been reduced proteolysis [10, 33, 34], improved glucose tolerance, improved caloric intake and weight gain [16, 24, 26-29, 31]. Negative reports of hyperinsulinaemia include hypoglycaemia and possible metabolic acidosis due to excessive carbohydrate oxidation [34, 35].

Persistent low blood glucose concentrations can reduce cerebral development and lead to long-term neurological deficiencies [36]. The upper limit for clinically desirable blood glucose concentration is also subject to debate [11]. Thus, glucose management goals vary widely between insulin therapy studies [30, 35] and it is likely the desired target range for glucose may change in the future. Similarly, Alsweiler et al [37] demonstrated a wide range of responses from different clinical units when questioned on glycaemic control targets. Hence, a control system needs to handle different glucose targets to comply with local practices. 
It is well known that neonatal response to insulin and glucose infusions exhibits great heterogeneity [11, 24], which would render fixed protocols ineffective because they do not adopt well to different patients or the evolution of metabolic response over time within an individual patient. Model-based blood glucose control may provide more optimized care by adapting in real-time to identified parameters representing the current metabolic state of the infant, and using this information to drive insulin dosing. This metabolic information can be combined with a controller utilising model predictions to achieve targeted blood glucose control. This approach has been validated in adult critical care studies [38]. However, sudden changes in patient condition independent of metabolic state indicate limits on model-based controller actions are required to maximise safety and control performance [39]. Insulin sensitivity changes can be captured and characterised using stochastic models of insulin sensitivity variability [40-42], specifically to quantify the level of hypoglycaemia risk and adjust control actions accordingly. This approach creates a targeted, model-based control system that uses stochastic forecasting to guarantee the risk of hypoglycaemia for any intervention.

Virtual trials offer the opportunity to explore control strategies in simulation to achieve the goals of maximising time within a desired glycaemic control band, which may vary between clinical units, whilst minimizing the number of hypoglycaemic episodes [38, 39]. Long-term clinical usage also requires robustness to missed BG measurements, control interventions, and delays in adjusting insulin dosing. The model-based controller used in this study tracks insulin sensitivity in real-time by fitting to available clinical data, and these errors may thus cause insulin dosing to be driven by an estimate of insulin sensitivity based on artificial effects rather than physiology. The effect of these errors can be evaluated in a clinically validated simulation environment to assess performance prior to clinical implementation. Short-term clinical trials are used to confirm model prediction accuracy and control efficacy. Pilot long-term clinical trials are presented to assess the agreement between simulation results and real-world outcomes, as well as to highlight some of the difficulties 
experienced in transitioning from the fully-compliant simulation environment to real-world clinical usage. 


\section{METHODS}

\subsection{System model}

The model is based on a clinically validated adult critical care glycaemic model, adapted to account for the main physiological differences in neonates.

$$
\begin{aligned}
\dot{G}= & -p_{G} \cdot G-S_{I} \cdot G \cdot \frac{Q}{1+\alpha_{G} Q} \\
& +\frac{P(t)+\left(P_{E N D} \cdot m_{\text {body }}\right)-\left(C N S \cdot m_{\text {brain }}\right)}{\left(V_{G, \text { frac }}(t) \cdot m_{\text {body }}\right)} \\
\dot{Q}= & -k Q+k I \\
\dot{I}= & -\frac{n I}{1+\alpha_{I} I}+\frac{u_{e x}(t)}{\left(V_{I, \text { frac }} \cdot m_{\text {body }}\right)}+e^{-\left(k_{1} u_{e x}(t)\right)} I_{B}
\end{aligned}
$$

Where $G(t)[\mathrm{mmol} / \mathrm{L}]$ is the total plasma glucose and $I(t)[\mathrm{mU} / \mathrm{L}]$ is the plasma insulin, exogenous insulin input is represented by $u_{e x}(\mathrm{t})[\mathrm{mU} / \mathrm{min}]$ and basal endogenous insulin secretion $I_{B}$ [mU/L/min], with $k_{I}$ representing the suppression of basal insulin secretion in the presence of exogenous insulin. The effect of previously infused insulin being utilised over time is represented by $Q(t)[\mathrm{mU} / \mathrm{L}]$, with $k\left[\mathrm{~min}^{-1}\right]$ accounting for the effective life of insulin in the system. Body weight and brain weight are denoted by $m_{\text {body }}[\mathrm{kg}]$ and $m_{\text {brain }}[\mathrm{kg}]$ respectively. Patient endogenous glucose clearance and insulin sensitivity are $p_{G}\left[\mathrm{~min}^{-1}\right]$ and $S_{I}[\mathrm{~L} /(\mathrm{mU} \cdot \mathrm{min})]$, respectively. The parameter $V_{I, f r a c}[\mathrm{~L} / \mathrm{kg}]$ is the insulin distribution volume per kilogram body weight and $n$ [ $\left.\min ^{-1}\right]$ is the constant first order decay rate for insulin from plasma. Total plasma glucose input is denoted $P(t)[\mathrm{mmol} / \mathrm{min}]$, endogenous glucose production is denoted by $P_{E N D}[\mathrm{mmol} / \mathrm{kg} / \mathrm{min}]$ and $V_{G, f r a c}$ [L/kg] represents the glucose distribution volume per kilogram of body weight. CNS [mmol/kg/min] represents non-insulin mediated glucose uptake by the central nervous system, as well as the liver, kidneys and red blood cells. Michaelis-Menten functions are used to model saturation, with $\alpha_{I}$ $[\mathrm{L} / \mathrm{mU}]$ used for the saturation of plasma insulin disappearance, and $\alpha_{G}[\mathrm{~L} / \mathrm{mU}]$ for the saturation of insulin-dependent glucose clearance. 
The insulin sensitivity parameter, $S_{I}$, drives the dynamics of the blood glucose model and is assumed patient-specific and independent of exogenous insulin and nutrition administration [38, 43]. Hourly insulin sensitivity is determined by fitting the model to retrospective data using integral-based fitting [44]. Once a patient-specific profile of time-varying insulin sensitivity is generated, it can be used to predict blood glucose concentration based on different insulin and nutrition control schemes. Such analyses are effectively in-silico or virtual trials [38, 43]. Overall median model BG forecast performance for 1, 2, 3 and 4-hour prediction windows are 5.2\%, 9.4\%, $11.9 \%$ and $13.6 \%$ respectively on retrospective data from 25 patients representing 3,567 hours of patient data [45]. The overall virtual trial development approach has been validated in the outcome of large scale trials [46].

\subsection{Stochastic model}

A two-dimensional kernel density estimation method is used to construct the stochastic model that describes the hour-to-hour variations in insulin sensitivity. The kernel density method combines probability distribution functions for each point of data representing change in insulin sensitivity from hour $n$ to hour $n+1$ to generate an overall density function for the data set. This produces a smooth, physiologically likely, continuous function across the parameter range [40-42]. The goal of this statistical model is to quantify the range of insulin sensitivity one hour ahead in time $\left(S_{I, n+1}\right)$ based on available data $\left(S_{I, n}, S_{I, n-1}, S_{I, n-2}, \ldots, S_{I, 0}\right)$ to guide real-time clinical control. The stochastic model forecasts quantify the upper and lower bound of likely future insulin sensitivity. These bounds are simulated using Equations (1)-(3) to generate upper and lower bounds for any future BG concentration and insulin intervention. Hence, these bounds are used by the controller to quantify the risk a selected insulin infusion rate will cause a hypoglycaemic measurement. If a hypoglycaemic event is forecasted the insulin rate may be reduced to eliminate the risk. Further details on stochastic model development and implementation in BG control are available in [40-42]. 


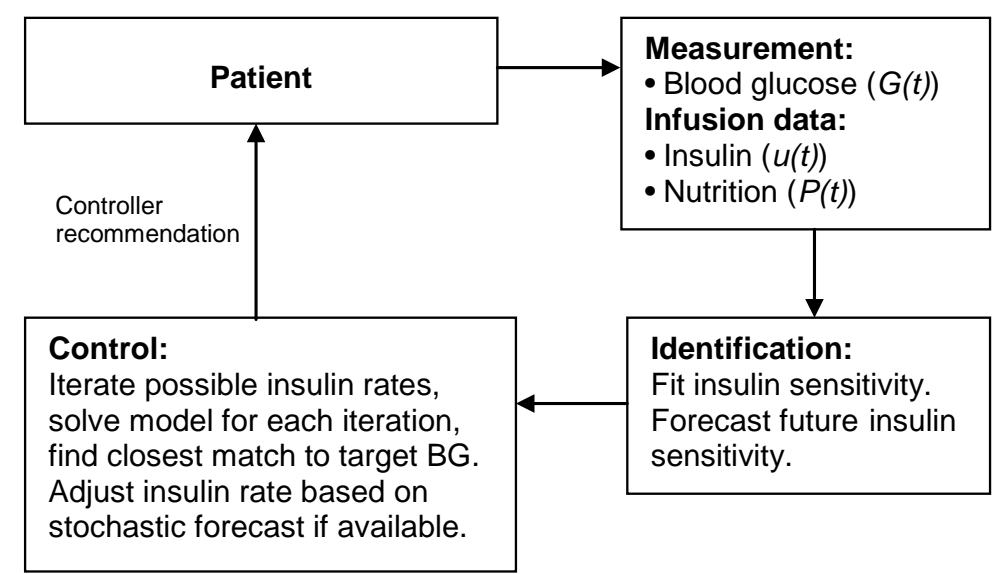

Figure 1: Controller implementation schematic

\subsection{Controller development}

The clinical implementation procedure for the controller is shown in Figure 1. The blood glucose history, together with insulin and nutrition history, is used to fit the patient's insulin sensitivity profile in real-time. This profile is then used by the controller to solve Equations (1)-(3) to predict blood glucose concentration based on insulin and nutrition rates. Thus, the controller adapts to the current metabolic state of the neonate in real-time. This control-scheme represents a semi-closed loop approach in that clinical staff oversee data entry into the control system and approve all insulin recommendations.

Sensitivity of the control scheme to BG measurement and control intervention frequency and BG sensor errors are explored in [39]. In this article, the following clinical considerations are explored:

- Clinical BG target range. The model-based controller was trialled in simulation with targets of $4 \mathrm{mmol} / \mathrm{L}, 5 \mathrm{mmol} / \mathrm{L}, 6 \mathrm{mmol} / \mathrm{L}, 7 \mathrm{mmol} / \mathrm{L}$ and $8 \mathrm{mmol} / \mathrm{L}$, which represents a range of target glucose concentrations likely to be encountered in neonatal critical care [37]. 
The lower acceptable BG forecast bound for an insulin intervention was set to $3 \mathrm{mmol} / \mathrm{L}$ for the $4 \mathrm{mmol} / \mathrm{L}$ target, $3.5 \mathrm{mmol} / \mathrm{L}$ for the $5 \mathrm{mmol} / \mathrm{L}$ target and $4 \mathrm{mmol} / \mathrm{L}$ for all other targets.

- Clinical intervention delays. Due to the spontaneous nature of emergency child-birth, doctors are often away from the unit assisting with a delivery. Additionally, nursing staff may be unavailable due to clinical instability with either the patient receiving insulin or another patient within the unit that requires immediate attention. Thus, substantial delays can exist between the time of BG measurement and eventual approval and adjustment of insulin infusion rate by clinical staff. The effect of these delays are modelled by incorporating a lag between the time of BG measurement and the time of insulin infusion change. Delays of an added 10, 20, 30 and 40 minutes based on experience were assessed.

- Missed BG measurement. A missed BG measurement and control intervention cycle may occur for reasons related to clinical workload, similar to the case of a delayed intervention. Control simulations were performed with a random missed BG measurement chance of 1/3, 1/4, 1/5 and 1/6 from a base measurement frequency of 2-hourly. These proportions of missed BG measurements (17\% - 33\% of measurements) are likely to be much higher than typical clinical practice, and thus represent a worst-case scenario test.

All simulations were performed with an assumed 7\% normally distributed BG sensor error and 2hourly BG measurement and intervention schedules. The retrospective clinically specified dextrose administration profile was used in all simulations to replicate the initial clinical use of this control system to only modulate the insulin side of the glucose-insulin balance. 


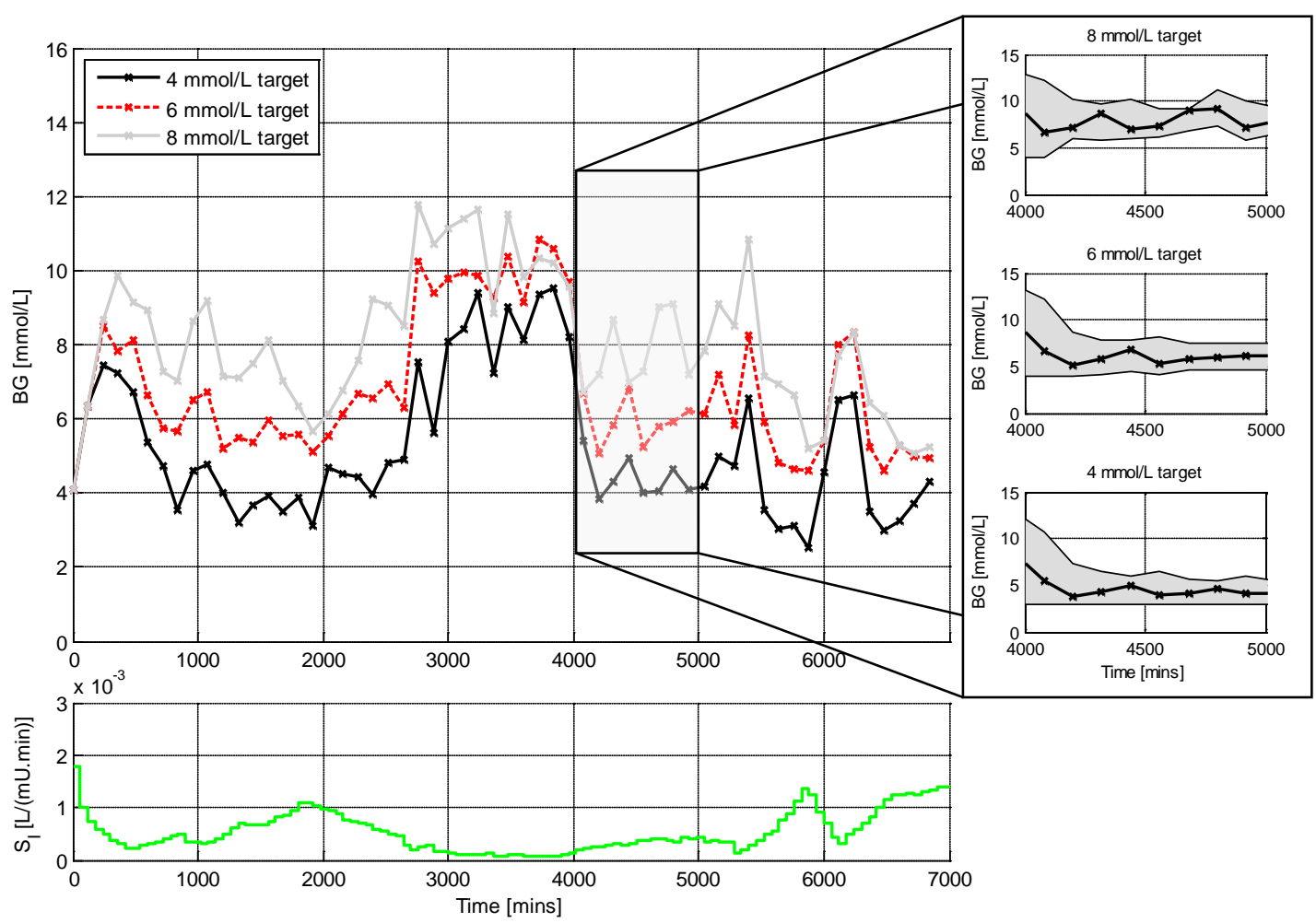

Figure 2: Retrospective patient profile with simulated controllers targeting $4 \mathrm{mmol} / \mathrm{L}, 5 \mathrm{mmol} / \mathrm{L}$ and $6 \mathrm{mmol} / \mathrm{L}$. The top panel shows the simulated BG response generated using each controller. The bottom panel shows the identified insulin sensitivity profile for this virtual patient.

\subsection{Patient cohort}

Retrospective data for 25 episodes of insulin usage over 21 patients from the Christchurch Women's Neonatal Intensive Care Unit was used in the study. Ethics approval for the clinical trials and publication of data was obtained from the Upper South Regional Ethics Committee. Median gestational age at birth was 26.6 weeks, and median birth weight was $0.845 \mathrm{~kg}$. Inclusion criteria were a period of treatment with insulin and at least six blood glucose measurements per day. Hourly-varying insulin sensitivity was fitted to each of the 25 patient profiles to generate a cohort of 25 'virtual patients' used for simulation. Further clinical details on the patient cohort and insulin sensitivity fitting procedure are available in [39, 45]. 
Two short-term pilot clinical trails (24 hours length each) are presented. These trials were conducted to assess model accuracy and control efficacy and were run by additional specialists to avoid increasing the technical burden on clinical staff. The two short-term trial patients were born at 27.3 and 27.0 weeks gestational age and weighted 770 grams and 900 grams at birth respectively. Additionally, two long-term (greater than 24 hours in length) pilot clinical trials of the model-based controller, demonstrating typical clinical usage, are also presented. The two long-term subjects (LT5 and LT4) were born at 25.0 weeks and 27.0 weeks gestational age respectively, with birth weight $0.530 \mathrm{~kg}$ and $0.870 \mathrm{~kg}$. The trials were conducted at age 22 days and 6 days respectively.

\section{RESULTS}

\section{Virtual trial simulations}

Figure 2 presents an example simulated trial employing controllers that target $4 \mathrm{mmol} / \mathrm{L}, 5 \mathrm{mmol} / \mathrm{L}$ and $6 \mathrm{mmol} / \mathrm{L}$ for a sample virtual patient. Each controller achieves BG control centred on the target band, and deviations from target are experienced during periods of low insulin sensitivity, such as shown in the period of 3,000 to 4,000 minutes in Figure 2, and during relatively rapid changes in glycaemic response to insulin as captured in the period of 5,500 to 6,500 minutes. The inset plots of Figure 2 show the shaded stochastic forecast bounds for glucose concentration for each controller. In the case of the $4 \mathrm{mmol} / \mathrm{L}$ target controller the stochastic forecast lower bound $\left(5^{\text {th }}\right.$ percentile) was uniformly at the set value of $3 \mathrm{mmol} / \mathrm{L}$ ensuring a guaranteed risk of no greater than $5 \%$ of exceeding this level. Thus, the requirement to maintain a low risk of a hypoglycaemic episode drove the insulin rates determined by the controller.

A comparison of BG control performance for the whole virtual patient cohort for different controller targets is presented in Table 1, and the cumulative distribution functions for BG 
concentration presented in Figure 3. The controller achieved a median BG within 1-5\% of target for all cases assessed. The stochastic model lower bound was lowered to $3 \mathrm{mmol} / \mathrm{L}$ and $3.5 \mathrm{mmol} / \mathrm{L}$ for the $4 \mathrm{mmol} / \mathrm{L}$ and $5 \mathrm{mmol} / \mathrm{L}$ targets respectively. The proportion of simulated BG measurements below the $3 \mathrm{mmol} / \mathrm{L}$ limits for the $4 \mathrm{mmol} / \mathrm{L}$ target controller was $4.7 \%$, and $3.3 \%$ of simulated $\mathrm{BG}$ measurements were below $3.5 \mathrm{mmol} / \mathrm{L}$ for the $5 \mathrm{mmol} / \mathrm{L}$ target controller. The remaining $\mathrm{BG}$ target controllers used $4 \mathrm{mmol} / \mathrm{L}$ as the lower bound for stochastic forecasts. The proportion of BG less than $4 \mathrm{mmol} / \mathrm{L}$ decreases for increasing BG target concentration as more insulin interventions forecasts have a lower bound above the $4 \mathrm{mmol} / \mathrm{L}$ cut-off, and thus the insulin infusion rate is not influenced by the use of the stochastic model for hypoglycaemia protection. In all cases, the percentage below the threshold is less than the guaranteed maximum risk of $5 \%$.

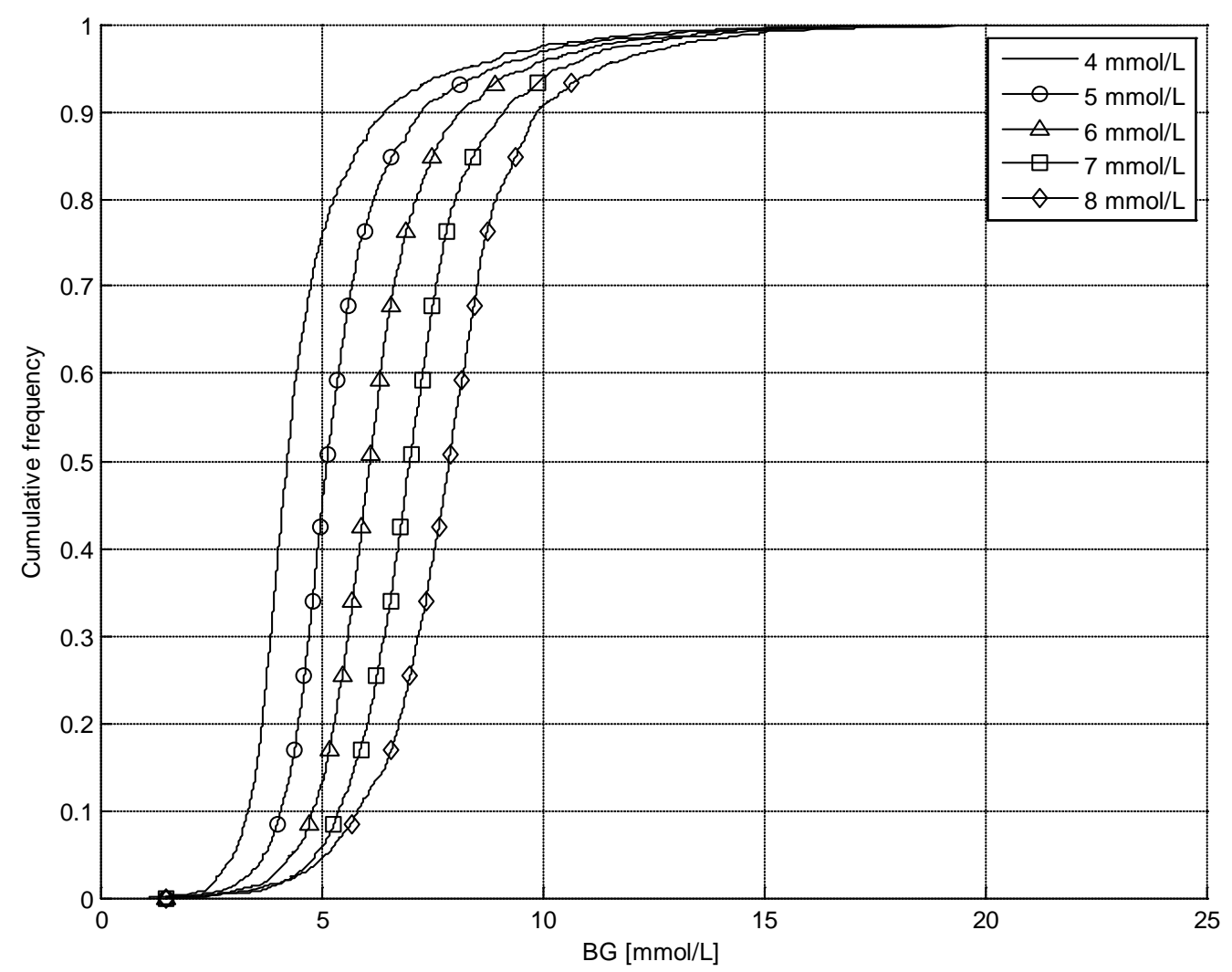

Figure 3: Empirical CDFs of model-based controller results incorporating BG targets of 4-8 $\mathrm{mmol} / \mathrm{L}$. The median value at 0.5 on the $\mathrm{y}$-axis for each target is within $1-5 \%$ in each case (see Table 1). 
The results for incorporating delays in BG measurement and clinical intervention are presented in Table 2 and Figure 4. These results confirm the model-based controller is robust to delays in insulin infusion rate changes, tending towards marginally higher proportion of hyperglycaemic measurements with longer delays. The stochastic model is thus a source of protection against significant increases in low BG measurements. Thus, the lower limits of the distributions presented in Figure 4 are essentially identical.

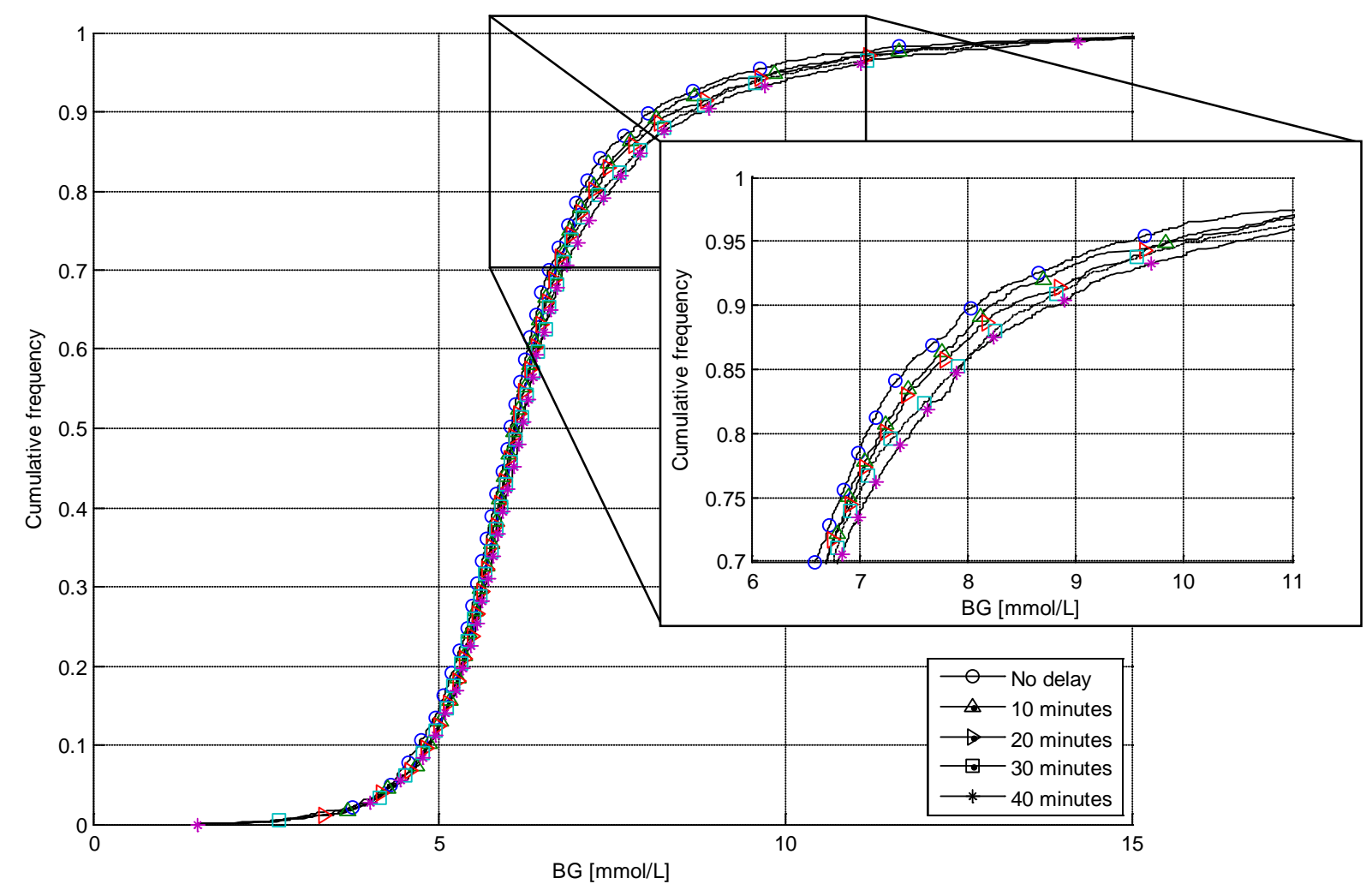

Figure 4: Empirical CDFs of model-based controller results incorporating delays between BG measurement and change of insulin infusion rate of $10-40$ minutes. The inset image highlights a region of the CDFs.

A comparison of BG control performance for simulations of missed BG measurement/control intervention cycles is presented in Table 3 and Figure 5. The missed BG measurements extend the average measurement frequency from the base value of 2 hours to $2.3-2.6$ hours. A general decrease in BG control quality is evident with increased IQR for increasing frequency of missed BG 
measurements, together with less measurements in the $4-7 \mathrm{mmol} / \mathrm{L}$ range and a greater proportion of measurements in the hypoglycaemic BG range $(<2.6 \mathrm{mmol} / \mathrm{L})$.

\section{Pilot clinical trials}

Table 4 and Figures 6 and 7 present short-term clinical trials of 24 hours each and long-term clinical trials covering 175.4 hours of stochastic targeted model-based BG control. The short-term trials achieved median BG concentrations of $5.7 \mathrm{mmol} / \mathrm{L}$ and $6.5 \mathrm{mmol} / \mathrm{L}$, noting that the first 4-6 hours in each case was devoted to reducing BG from hyperglycaemic levels. BG prediction accuracy in response to an intervention was $8.5 \%$ - $8.6 \%$ for a median measurement period of 2.0 hours. The stochastic model forecasts captured 31 - 50\% of BG values within the IQR and 83 - 85\% within the $5 \%$ - 95\% confidence interval.

The long-term trials were conducted by clinical staff using the model-based control software. BG prediction accuracy in response to an intervention for LT5 was $14.4 \%$ for a median BG measurement period of 3.3 hours. Median BG concentration was $6.0 \mathrm{mmol} / \mathrm{L}$, with $68.3 \%$ of measurements within the $4-7 \mathrm{mmol} / \mathrm{L}$ band. The stochastic model forecast coverage captured $28 \%$

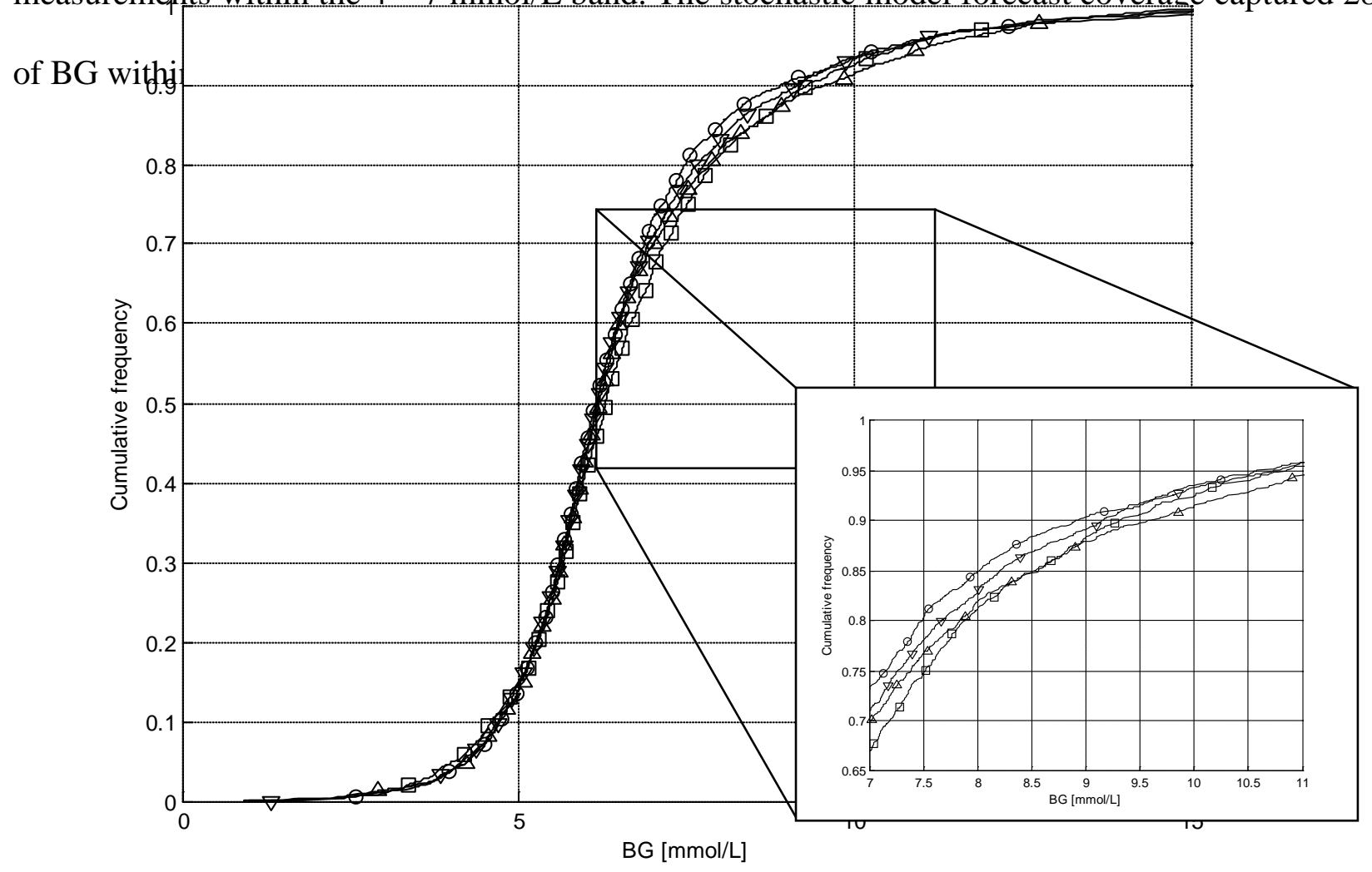


Figure 5: Empirical CDFs of model-based controller results for simulated missed BG measurements. The inset image highlights a portion of the CDF curves for clarity.

Clinical implementation errors were experienced during long-term trial LT4. Overall, 18\% and 27\% of BG forecasts were within the IQR and 5\%-95\% probability bounds. Only $14.3 \%$ of measurements were within the $4-7 \mathrm{mmol} / \mathrm{L}$ range. Figure 7 shows the effect of a BG measurement that was not entered into the control software. At 566 minutes into the trial a BG measurement of $3.5 \mathrm{mmol} / \mathrm{L}$ was measured but not entered. This missing measurement caused the following sequence of events:

a) BG measurement of $3.5 \mathrm{mmol} / \mathrm{L}$ at 566 minutes caused insulin to be shut off. None of this data was entered into the computer.

b) Measurement of BG at 781 minutes was $10.3 \mathrm{mmol} / \mathrm{L}$ and entered into computer.

c) The controller believed that:

i) Insulin was still running over the period of (a) to (b)

ii) The BG measurement of $3.5 \mathrm{mmol} / \mathrm{L}$ never occurred, and

iii) BG had risen steadily over the last several hours despite constant insulin infusions.

d) Hence, an artificially low insulin sensitivity profile, shown as the dashed line on the bottom panel of Figure 7, was fitted based on available data.

e) An increased insulin rate of $0.48 \mathrm{~mL} / \mathrm{hr}$ was thus recommended by controller, whereas if all data was present an insulin rate of $0.12 \mathrm{~mL} / \mathrm{hr}$ would have been selected. This may have contributed to the low BG measurement at 896 minutes. 
A filter to block air bubbles from reaching the patient was present in the insulin line for this patient. Clinical protocol is to avoid filters in lines delivering insulin as this can impede insulin delivery. The filter was removed at approximately 2,500 minutes, which corresponded with a rapid rise in insulin sensitivity and subsequently much lower insulin infusion requirements for this patient.
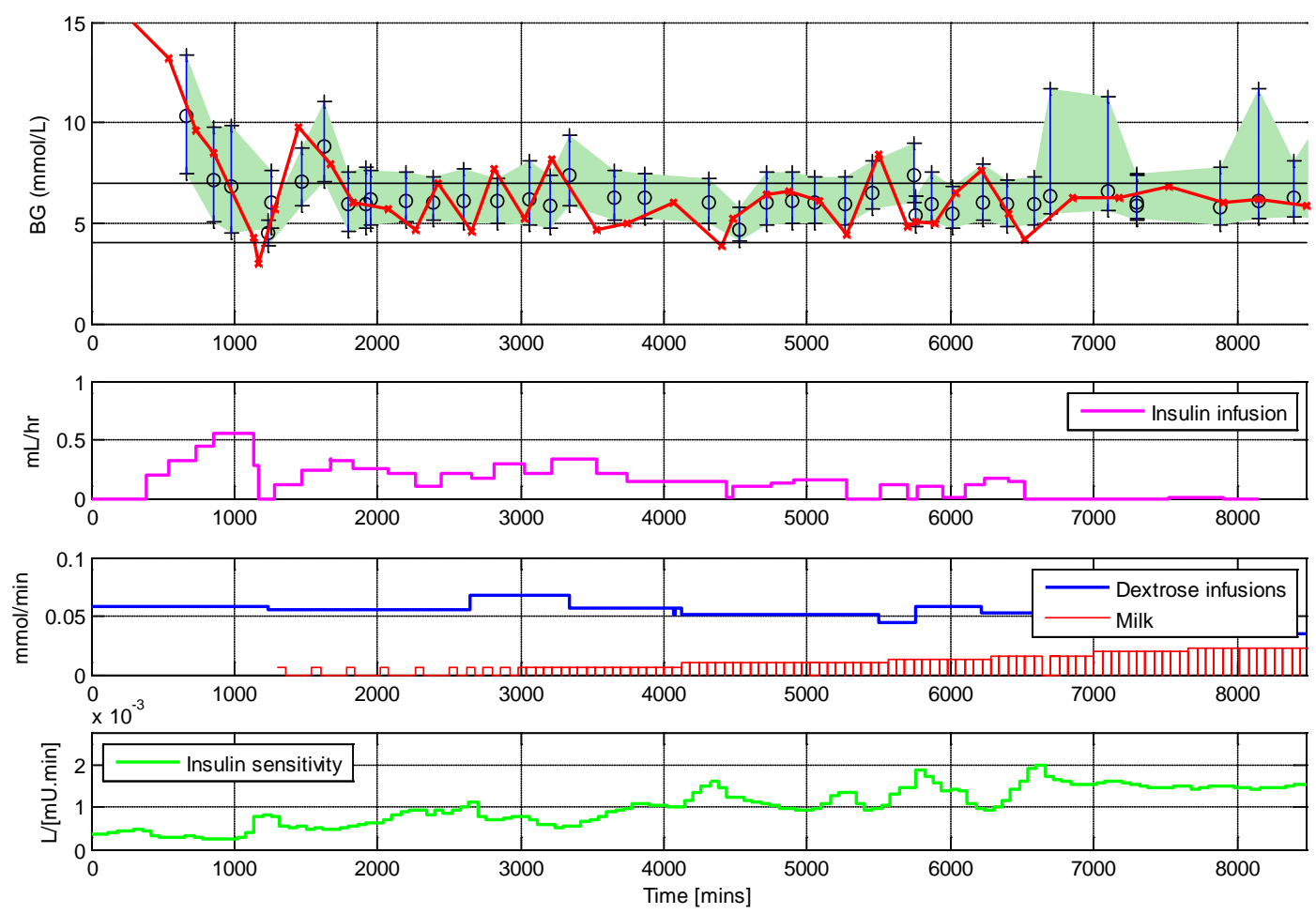

Figure 6: Long-term glycaemic control for patient LT5. The top panel shows measured BG concentration and the shaded areas represent the forecasted 5\%-95\% range generated during realtime control. The second panel displays the insulin infusion rate, and the third panel shows parenteral dextrose infusion (solid line) and breast milk feedings (bars). The bottom panel shows model-fitted insulin sensitivity. 


\section{DISCUSSION}

Real-time model-based glucose control can offer advantages over fixed protocols by accounting for inter-patient responses to insulin infusions and track metabolic response over time. The use of retrospective data to generate virtual patients allows investigations and refinements of control strategies in simulation before initial pilot trials. Initial short-term trials up to 24 hours where the controller was run by extra specialists were very successful and similar to expected results from virtual trials, as shown in Table 4 and [39].

However, translating long-term (greater than 24 hours) glycaemic control from simulation and shorter trials to the clinical world and direct clinical use requires accounting for clinical and system errors. In addition, the approach to design and test control in simulation before implementation is a novel approach towards glycaemic control in neonatal care. In particular, the extreme fragility and low blood volumes of these patients means many standard metabolic tests, such as IVGTTs and tracer studies are both technically and ethically difficult.

Figure 3 shows the different distributions of simulated BG measurements have largely the same shape, but are shifted for different targets. Model-based control can be set to target a specific glucose concentration relatively easily. This ease and directness in setting a target is in contrast with fixed sliding scale or similar control approaches that must be completely re-calibrated for each target and patient. A possible modification to a fixed protocol to target a different glycaemic level may be to uniformly increase insulin infusion rates. However, this may increase the risk of hypoglycaemic for more insulin-sensitivity patients, and can lead to a negative study result, whereas the inability of the underlying protocol to adequately address inter-patient differences may be an underlying cause. 
Blood glucose control targets in model-based control are customisable to within a reasonable range. Very low targets run higher risks of hypoglycaemic measurements and are negated by the use of stochastic model forecasting in the control algorithm. Very high targets cannot be achieved without increasing dextrose infusions. Clinicians may also select a BG target based on emerging research in this field, or on a per-patient basis. This target setting feature may be useful in research settings to elucidate the specific effects of glucose control to a range of targets on neonatal outcome. Additionally, specific nutrition goals may be set per-patient with the controller adjusting insulin to maintain overall metabolic balance.

The missed BG measurements and clinical interventions delays simulated in this study represent a sample of the possible clinical consequences that may be captured. The probability of a missed measurement was assumed uniformly distributed in this study, where in reality the chance of successive BG measurements being missed is likely lower. Thus, the results presented in Table 3 reflect a more rigorous stress-test of protocol robustness. More realistic probabilities of missed measurements could be captured by more detailed models, and allow the control designer a choice between testing “robustness" and "reality” for a proposed control system.

Insulin sensitivity in this model represents the net whole-body response to glucose and insulin inputs. Its variation may be due to physiological factors such as medications, stress and/or clinical condition. However, importantly, the apparent insulin sensitivity may also be influenced by technical concerns, such as filters in the insulin line which can restrict insulin delivered to the patient. Incomplete data may also have an effect, as demonstrated in Figure 7. Fully closed-loop control may mitigate some of the errors assessed in this study, but can also introduce new sources of 
system failure such as sensor failure, calibration drift and communication failures between the sensor, control system and/or pump. Thus, the use of simulation tools to assess the magnitude of consequences before clinical implementation is applicable to either control strategy.

In this study, compartment models were used for control as opposed to "black-box" purely datadriven modelling techniques [47]. The use of a physiologically-based model may afford a clinical view into specific mechanisms of metabolic disturbances in this population. Disturbances in modelfitted insulin sensitivity may be linked to clinical condition and, in one adult ICU study, the evolution of sepsis [48]. Given the relatively recent advancements in neonatal intensive care that allow more extremely premature infants to survive, much is still unknown about the ontogeny of neonatal metabolic control systems and such model-based analysis may shed added light.

The controller attempts to lower BG concentration through steady reductions in BG level, rather than trying to reach the target range as quickly as possible [39]. Limits on insulin infusion rate changes for the controller were explored and showed that gradual changes in insulin infusion rate provided an optimal trade-off between the amount of BG measurements and risk of hypoglycaemic episodes [39]. Thus, it is likely some measure of robustness of the controller can be attributed to reduced accumulated errors, as the change in insulin infusion rate between successive interventions is generally small.

Stochastic model forecast bounds are used by the controller to limit the risk of hypoglycaemia or excursions below any threshold. Data on insulin sensitivity variation for a whole cohort is used to generate the stochastic model used in this study. Thus, the model forecast bounds would be expected to adequately cover BG predictions over the whole cohort. However, they may equally 
well be over or under- conservative for any particular patient, as demonstrated in Table 4 by the differences in stochastic model performance for patients LT1 and LT4.

Figure 2 shows the stochastic bounds for a virtual trial at three different BG targets. For lower BG targets, the lower bound of forecasted BG concentration is used more often as the criteria for the selection of insulin rates. Thus, there is interplay between the target glucose concentration and risk of hypoglycaemia, which can be both quantified and evaluated in simulation using retrospective data.

Further improvements to the model and controller could incorporate daily nutritional and volume goals that can be set by clinicians with model-based targeted control taking care of glycaemia - thus relieving clinical staff from estimation and ad-hoc decision making. The ideal content and composition of nutritional regimes for preterm infants is still under debate. The proportions of dextrose, protein and lipids given in the NICU may be different to what an infant receives in-utero. Whilst the relevant major organs express many of the biological mechanisms responsible for glucose regulation from a relatively early age, the foetus depends upon the mother to control energy supply. Thus, the controller is essentially attempting to replicate some of the mother's functions, as well as account for the synchronised processes that regulate foetal growth that are perturbed by premature birth and life outside the womb. 

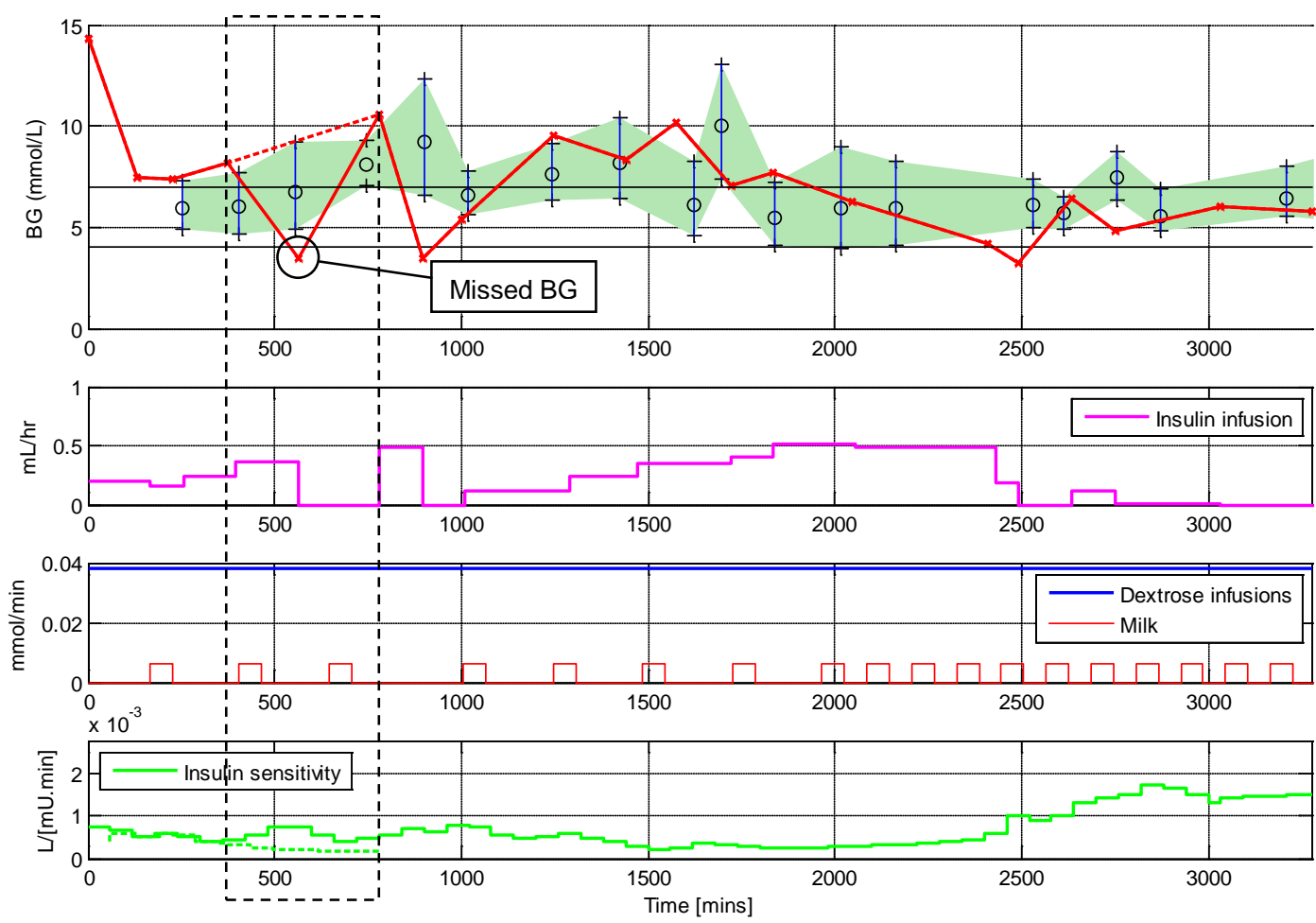

Figure 7: Long-term glycaemic control for patient LT4. The top panel shows measured BG concentration and the shaded areas represent the forecasted 5\%-95\% range generated during real-time control. The second panel displays the insulin infusion rate, and the third panel shows parenteral dextrose infusion (solid line) and breast milk feedings (bars). The bottom panel shows insulin sensitivity as fitted post-trial. The dashed lines for blood glucose and insulin sensitivity show the effect of missing data entry for the BG measurement at 566 minutes (circled). This missing measurement caused the observed insulin sensitivity to be lower than the correct fitted value, thus the controller chose a higher insulin rate contributing to a low BG measurement at 781 minutes. The rapid rise in insulin sensitivity beginning at approximately 2,500 minutes corresponds with the removal of a filter from the insulin line.

\section{CONCLUSIONS}

A model of the neonatal glucose regulatory system is used to design controllers for long-term clinical usage in neonatal care. Simulated trials revealed the sensitivity of control performance to missed BG measurements and delays in implementing interventions. Control schemes targeting a range of BG concentrations indicated an ability to customise control for a particular neonatal unit's practices. Pilot long-term trials highlighted the difficulties sometimes encountered in translating a control system from simulations and highly controlled pilot trials to real-world everyday clinical usage. In particular, the fidelity of translating both BG measurements and resulting insulin interventions to the model-based controller can present a significant challenge that must be addressed by appropriate system software design. 


\section{Conflict of interest statement}

The authors state they have no outstanding conflicts of interest.

\section{Financial Support}

New Zealand Tertiary Education Commission. This funding source had no role in the study design, collection, analysis or interpretation of the data, in the writing of the manuscript or the decision to submit the manuscript.

\section{References}

[1] H. S. Dweck and G. Cassady, "Glucose Intolerance in Infants of Very Low Birth Weight," Pediatrics, vol. 53, pp. 189-195, 1974.

[2] L. S. Kao, B. H. Morris, K. P. Lally, C. D. Stewart, V. Huseby, and K. A. Kennedy, "Hyperglycemia and morbidity and mortality in extremely low birth weight infants," Journal of perinatology, vol. 26, pp. 7306, 2006.

[3] S. P. Hays, B. Smith, and A. L. Sunehag, "Hyperglycemia Is a Risk Factor for Early Death and Morbidity in Extremely Low Birth-Weight Infants," Pediatrics, vol. 118, pp. 1811-1818, 2006.

[4] K. Heimann, T. Peschgens, R. Kwiecien, S. Stanzel, H. Hoernchen, and U. Merz, "Are recurrent hyperglycemic episodes and median blood glucose level a prognostic factor for increased morbidity and mortality in premature infants </=1500 g?," Journal of perinatal medicine, vol. 35, pp. 245-8, 2007.

[5] N. J. Hall, M. Peters, S. Eaton, and A. Pierro, "Hyperglycemia is associated with increased morbidity and mortality rates in neonates with necrotizing enterocolitis," Journal of pediatric surgery, vol. 39, pp. 898-901, 2004.

[6] G. B. Avery, M. A. Fletcher, and M. G. MacDonald, Neonatology : pathophysiology and management of the newborn., 4th ed: Philadelphia : Lippincott, 1994.

[7] T. Ertl, J. Gyarmati, V. Gaal, and I. Szabo, "Relationship between hyperglycemia and retinopathy of prematurity in very low birth weight infants," Biology of the neonate, vol. 89, pp. 56-9, 2006.

[8] R. Garg, A. G. Agthe, P. K. Donohue, and C. U. Lehmann, "Hyperglycemia and retinopathy of prematurity in very low birth weight infants," Journal of perinatology, vol. 23, pp. 186-94, 2003.

[9] D. I. Alaedeen, M. C. Walsh, and W. J. Chwals, "Total parenteral nutrition-associated hyperglycemia correlates with prolonged mechanical ventilation and hospital stay in septic infants," J Pediatr Surg, vol. 41, pp. 239-44, 2006.

[10] M. S. Agus, P. J. Javid, D. P. Ryan, and T. Jaksic, "Intravenous insulin decreases protein breakdown in infants on extracorporeal membrane oxygenation," Journal of pediatric surgery, vol. 39, pp. 839-44, 2004.

[11] R. M. Cowett and H. M. Farrag, "Selected principles of perinatal-neonatal glucose metabolism," Semin Neonatol, vol. 9, pp. 37-47, 2004.

[12] D. Mitanchez-Mokhtari, N. Lahlou, F. Kieffer, J.-F. Magny, M. Roger, and M. Voyer, "Both Relative Insulin Resistance and Defective Islet \{beta\}-Cell Processing of Proinsulin Are Responsible for Transient Hyperglycemia in Extremely Preterm Infants," Pediatrics, vol. 113, pp. 537-541, 2004.

[13] R. M. Cowett, W. Oh, and R. Schwartz, "Persistent glucose production during glucose infusion in the neonate," J Clin Invest, vol. 71, pp. 467-75, 1983.

[14] M. Raney, A. Donze, and J. R. Smith, "Insulin infusion for the treatment of hyperglycemia in low birth weight infants: examining the evidence," Neonatal Netw, vol. 27, pp. 127-40, 2008.

[15] D. P. Barker and N. Rutter, "Stress, severity of illness, and outcome in ventilated preterm infants," Archives of disease in childhood, vol. 75, pp. F187-90, 1996.

[16] F. Thabet, J. Bourgeois, B. Guy, and G. Putet, "Continuous insulin infusion in hyperglycaemic very-lowbirth-weight infants receiving parenteral nutrition," Clin Nutr, vol. 22, pp. 545-7, 2003.

[17] A. H. Hemachandra and R. M. Cowett, "Neonatal Hyperglycemia," Pediatrics in Review, vol. 20, pp. 16e-24, 1999.

[18] S. L. Goldman and T. Hirata, "Attenuated response to insulin in very low birthweight infants," Pediatric research, vol. 14, pp. 50-3, 1980. 
[19] H. M. Farrag and R. M. Cowett, "Glucose homeostasis in the micropremie," Clinics in Perinatology, vol. 27, pp. 1-22, 2000.

[20] G. Hartnoll, "Basic principles and practical steps in the management of fluid balance in the newborn," Seminars in neonatology, vol. 8, pp. 307-13, 2003.

[21] R. Hume, A. Burchell, F. L. Williams, and D. K. Koh, "Glucose homeostasis in the newborn," Early Hum Dev, vol. 81, pp. 95-101, 2005.

[22] H. M. Farrag, L. M. Nawrath, J. E. Healey, E. J. Dorcus, R. E. Rapoza, W. Oh, and R. M. Cowett, "Persistent glucose production and greater peripheral sensitivity to insulin in the neonate vs. the adult," Am J Physiol, vol. 272, pp. E86-93, 1997.

[23] A. Natali, A. Gastaldelli, S. Camastra, A. M. Sironi, E. Toschi, A. Masoni, E. Ferrannini, and A. Mari, "Dose-response characteristics of insulin action on glucose metabolism: a non-steady-state approach," Am J Physiol Endocrinol Metab, vol. 278, pp. E794-801, 2000.

[24] J. W. Collins, Jr., M. Hoppe, K. Brown, D. V. Edidin, J. Padbury, and E. S. Ogata, "A controlled trial of insulin infusion and parenteral nutrition in extremely low birth weight infants with glucose intolerance," The Journal of pediatrics, vol. 118, pp. 921-7, 1991.

[25] K. Beardsall, S. Vanhaesebrouck, A. L. Ogilvy-Stuart, J. S. Ahluwalia, C. Vanhole, C. Palmer, P. Midgley, M. Thompson, L. Cornette, M. Weissenbruch, M. Thio, F. de Zegher, and D. Dunger, "A randomised controlled trial of early insulin therapy in very low birth weight infants, "NIRTURE" (neonatal insulin replacement therapy in Europe)," BMC pediatrics, vol. 7, pp. 29, 2007.

[26] W. Meetze, R. Bowsher, J. Compton, and H. Moorehead, "Hyperglycemia in extremely- low-birthweight infants," Biol Neonate, vol. 74, pp. 214-21, 1998.

[27] N. D. Binder, P. K. Raschko, G. I. Benda, and J. W. Reynolds, "Insulin infusion with parenteral nutrition in extremely low birth weight infants with hyperglycemia," The Journal of pediatrics, vol. 114, pp. 273-80, 1989.

[28] S. Ostertag, L. Jovanovic, B. Lewis, and P. Auld, "Insulin pump therapy in the very low birth weight infant," Pediatrics, vol. 78, pp. 625-630, 1986.

[29] Y. E. Vaucher, P. D. Walson, and G. Morrow, 3rd, "Continuous insulin infusion in hyperglycemic, very low birth weight infants," Journal of pediatric gastroenterology and nutrition, vol. 1, pp. 211-7, 1982.

[30] D. Vlasselaers, I. Milants, L. Desmet, P. J. Wouters, I. Vanhorebeek, I. van den Heuvel, D. Mesotten, M. P. Casaer, G. Meyfroidt, C. Ingels, J. Muller, S. Van Cromphaut, M. Schetz, and G. Van den Berghe, "Intensive insulin therapy for patients in paediatric intensive care: a prospective, randomised controlled study," Lancet, vol. 373, pp. 547-56, 2009.

[31] K. S. Kanarek, M. L. Santeiro, and J. I. Malone, "Continuous infusion of insulin in hyperglycemic lowbirth weight infants receiving parenteral nutrition with and without lipid emulsion," J Parenter Enteral Nutr, vol. 15, pp. 417-20, 1991.

[32] S. M. Ng, J. E. May, and A. J. Emmerson, "Continuous insulin infusion in hyperglycaemic extremelylow- birth-weight neonates," Biol Neonate, vol. 87, pp. 269-72, 2005.

[33] M. S. Agus, P. J. Javid, H. G. Piper, D. Wypij, C. P. Duggan, D. P. Ryan, and T. M. Jaksic, "The Effect of Insulin Infusion Upon Protein Metabolism in Neonates on Extracorporeal Life Support," Annals of Surgery Scientific Papers of the 126th Annual Meeting of the American Surgical Association, vol. 244, pp. 536-544, 2006.

[34] B. B. Poindexter, C. A. Karn, and S. C. Denne, "Exogenous insulin reduces proteolysis and protein synthesis in extremely low birth weight infants," The Journal of pediatrics, vol. 132, pp. 948-53, 1998.

[35] K. Beardsall, S. Vanhaesebrouck, A. L. Ogilvy-Stuart, C. Vanhole, C. R. Palmer, M. van Weissenbruch, P. Midgley, M. Thompson, M. Thio, L. Cornette, I. Ossuetta, I. Iglesias, C. Theyskens, M. de Jong, J. S. Ahluwalia, F. de Zegher, and D. B. Dunger, "Early Insulin Therapy in Very-Low-Birth-Weight Infants," N Engl J Med, vol. 359, pp. 1873-1884, 2008.

[36] A. Lucas, R. Morley, and T. J. Cole, "Adverse neurodevelopmental outcome of moderate neonatal hypoglycaemia," Br Med J, vol. 297, pp. 1304-1308, 1988.

[37] J. M. Alsweiler, C. A. Kuschel, and F. H. Bloomfield, "Survey of the management of neonatal hyperglycaemia in Australasia," Journal of Paediatrics and Child Health, vol. 43, pp. 632-635, 2007.

[38] J. G. Chase, G. M. Shaw, T. Lotz, A. LeCompte, J. Wong, J. Lin, T. Lonergan, M. Willacy, and C. E. Hann, "Model-based insulin and nutrition administration for tight glycaemic control in critical care," Curr Drug Deliv, vol. 4, pp. 283-96, 2007.

[39] A. Le Compte, J. Chase, A. Lynn, C. Hann, G. Shaw, X. Wong, and J. Lin, "Blood Glucose Controller for Neonatal Intensive Care: Virtual trials development and 1st clinical trials," Journal of Diabetes Science and Technology, vol. 3, pp. 1066-1081, 2009.

[40] J. Lin, D. Lee, J. Chase, C. Hann, T. Lotz, and X. Wong, "Stochastic Modelling of Insulin Sensitivity Variability in Critical Care," Biomedical Signal Processing \& Control, vol. 1, pp. 229-242, 2006.

[41] J. Lin, D. Lee, J. G. Chase, G. M. Shaw, A. Le Compte, T. Lotz, J. Wong, T. Lonergan, and C. E. Hann, "Stochastic modelling of insulin sensitivity and adaptive glycemic control for critical care," Computer Methods and Programs in Biomedicine, vol. 89, pp. 141-152, 2008. 
[42] A. Le Compte, D. Lee, J. G. Chase, J. Lin, A. Lynn, and G. Shaw, "Blood Glucose Prediction Using Stochastic Modeling in Neonatal Intensive Care," IEEE Trans Biomed Eng, vol. 57, pp. 509-518, 2009.

[43] T. Lonergan, A. LeCompte, M. Willacy, J. G. Chase, G. M. Shaw, X. W. Wong, T. Lotz, J. Lin, and C. E. Hann, "A simple insulin-nutrition protocol for tight glycemic control in critical illness: development and protocol comparison," Diabetes Technol Ther, vol. 8, pp. 191-206, 2006.

[44] C. E. Hann, J. G. Chase, J. Lin, T. Lotz, C. V. Doran, and G. M. Shaw, "Integral-based parameter identification for long-term dynamic verification of a glucose-insulin system model," Comput Methods Programs Biomed, vol. 77, pp. 259-270, 2005.

[45] A. Le Compte, "Modelling the Glucose-Insulin Regulatory System for Glycaemic Control in Neonatal Intensive Care," PhD thesis in Mechanical Engineering, University of Canterbury, Christchurch, New Zealand, http://ir.canterbury.ac.nz/.

[46] J. G. Chase, G. Shaw, A. Le Compte, T. Lonergan, M. Willacy, X.-W. Wong, J. Lin, T. Lotz, D. Lee, and C. Hann, "Implementation and evaluation of the SPRINT protocol for tight glycaemic control in critically ill patients: a clinical practice change," Critical Care, vol. 12, pp. R49, 2008.

[47] Z. Trajanoski and $P$. Wach, "Neural predictive controller for insulin delivery using the subcutaneous route," IEEE Trans Biomed Eng, vol. 45, pp. 1122-1134, 1998.

[48] A. Blakemore, S. Wang, A. Le Compte, G. Shaw, X. Wong, J. Lin, T. Lotz, C. Hann, and J. Chase, "Model-Based Insulin Sensitivity as a Sepsis Diagnostic in Critical Care," Journal of Diabetes Science and Technology, vol. 2, pp. 468-477, 2008.

Table 1: Glucose control performance for different BG targets for the model-based controller.

\begin{tabular}{|c|c|c|c|c|c|}
\hline \multirow{2}{*}{ Target BG } & \multicolumn{2}{|c|}{ BG (mmol/L) } & \multicolumn{2}{c|}{ \% of measurements within range } \\
\cline { 2 - 6 } & Median & IQR & $\mathbf{4 - 7} \mathbf{~ m m o l / L}$ & $<\mathbf{4 ~ m m o l / L}$ & $<\mathbf{2 . 6} \mathbf{~ m m o l} / \mathbf{L}$ \\
\hline $\mathbf{4} \mathbf{~ m m o l} / \mathbf{L}$ & 4.2 & {$[3.7-5.0]$} & 54.4 & 37.8 & 2.0 \\
\hline $\mathbf{5} \mathbf{~ m m o l} / \mathbf{L}$ & 5.1 & {$[4.6-5.9]$} & 79.7 & 8.3 & 0.6 \\
\hline $\mathbf{6 ~} \mathbf{~ m m o l} / \mathbf{L}$ & 6.0 & {$[5.4-6.8]$} & 75.6 & 3.2 & 0.3 \\
\hline $\mathbf{7 ~} \mathbf{~ m o l} / \mathbf{L}$ & 7.0 & {$[6.2-7.8]$} & 48.4 & 1.5 & 0.3 \\
\hline $\mathbf{8} \mathbf{~ m m o l} / \mathbf{L}$ & 7.9 & {$[7.0-8.7]$} & 23.7 & 1.5 & 0.3 \\
\hline
\end{tabular}

Table 2: Glucose control performance for delays between BG measurement and insulin infusion rate change.

\begin{tabular}{|c|c|c|c|c|c|}
\hline \multirow{2}{*}{ Delay } & \multicolumn{2}{|c|}{ BG (mmol/L) } & \multicolumn{3}{|c|}{$\%$ of measurements within range } \\
\hline & Median & IQR & $4-7 \mathrm{mmol} / \mathrm{L}$ & $<4 \mathrm{mmol} / \mathrm{L}$ & $<2.6 \mathrm{mmol} / \mathrm{L}$ \\
\hline None & 6.0 & {$[5.4-6.8]$} & 75.6 & 3.2 & 0.3 \\
\hline $10 \mathrm{~min}$ & 6.1 & {$[5.5-6.9]$} & 74.0 & 2.9 & 0.4 \\
\hline $20 \mathrm{~min}$ & 6.1 & {$[5.5-6.9]$} & 73.7 & 2.7 & 0.3 \\
\hline $30 \mathrm{~min}$ & 6.2 & {$[5.5-7.0]$} & 73.0 & 2.4 & 0.3 \\
\hline $40 \mathrm{~min}$ & 6.2 & {$[5.5-7.1]$} & 71.0 & 2.7 & 0.5 \\
\hline
\end{tabular}


Table 3: Glucose control performance and frequency of measurement versus change of a missed BG measurement.

\begin{tabular}{|c|c|c|c|c|c|}
\hline \multirow{2}{*}{$\begin{array}{c}\text { Chance of } \\
\text { missed } \\
\text { measurement }\end{array}$} & \multicolumn{2}{|c|}{ BG (mmol/L) } & \multicolumn{2}{c|}{ \% of measurements within range } \\
\cline { 2 - 6 } & Median & IQR & $\mathbf{4 - 7} \mathbf{~ m m o l / L}$ & $<\mathbf{4 ~ m m o l / L}$ & $<\mathbf{2 . 6} \mathbf{~ m m o l} / \mathbf{L}$ \\
\hline $\mathbf{1 / 3}$ & 6.3 & {$[5.5-7.5]$} & 62.1 & 4.8 & 0.7 \\
\hline $\mathbf{1 / 4}$ & 6.2 & {$[5.5-7.4]$} & 66.1 & 3.7 & 0.8 \\
\hline $\mathbf{1 / 5}$ & 6.2 & {$[5.5-7.2]$} & 69.2 & 3.9 & 0.6 \\
\hline $\mathbf{1 / 6}$ & 6.2 & {$[5.5-7.3]$} & 67.4 & 3.6 & 0.6 \\
\hline None & 6.0 & {$[5.4-6.8]$} & 75.6 & 3.2 & 0.3 \\
\hline
\end{tabular}

Table 4: Glycaemic control summary for pilot trials. Patients STB and STF were short-term pilot trials and patients LT5 and LT4 were long-term pilot trials.

\begin{tabular}{|c|c|c|c|c|}
\hline & \multicolumn{2}{|c|}{ Short-term trials } & \multicolumn{2}{|c|}{ Long-term trials } \\
\hline & STB & STF & LT5 & LT4 \\
\hline Birth weight & 770 grams & 900 grams & 530 grams & 870 grams \\
\hline Birth gestational age & 27.3 weeks & 27.0 weeks & 25.0 weeks & 27.0 weeks \\
\hline Age at start of trial & 9 days & 2 days & 22 days & 6 days \\
\hline Total hours of control & 24 hours & 24 hours & 141.3 hours & 34.1 hours \\
\hline BG median [IQR] & $5.7[5.4-7.2]$ & $6.5[4.7-8.2]$ & $6.0[5.0-7.6]$ & $7.6[6.3-9.5]$ \\
\hline$\%$ within $4-7 \mathrm{mmol} / \mathrm{L}$ range & $55.6 \%$ & $46.7 \%$ & $68.3 \%$ & $14.3 \%$ \\
\hline $\begin{array}{l}\text { Median hours between BG } \\
\text { [IQR] }\end{array}$ & $\begin{array}{c}1.8 \\
{[1.1-2.4]}\end{array}$ & $\begin{array}{c}2.0 \\
{[1.5-2.0]}\end{array}$ & $\begin{array}{c}3.3 \\
{[2.5-4.4]}\end{array}$ & $\begin{array}{c}2.4 \\
{[1.9-3.3]}\end{array}$ \\
\hline $\begin{array}{l}\text { Median BG forecast error } \\
\text { [IQR] }\end{array}$ & $\begin{array}{c}8.6 \% \\
{[3.4 \%-11.7 \%]}\end{array}$ & $\begin{array}{c}8.5 \% \\
{[4.9 \%-27.1 \%]}\end{array}$ & $\begin{array}{c}14.4 \% \\
{[4.2 \%-25.6 \%]}\end{array}$ & $\begin{array}{c}27.4 \% \\
{[20.1 \%-34.2 \%]}\end{array}$ \\
\hline $\begin{array}{l}\% \text { forecasts within IQR } \\
\text { predictions }\end{array}$ & $31 \%$ & $50 \%$ & $28 \%$ & $18 \%$ \\
\hline $\begin{array}{l}\% \text { forecasts within } 5 \%-95 \% \\
\text { predictions }\end{array}$ & $85 \%$ & $83 \%$ & $64 \%$ & $27 \%$ \\
\hline
\end{tabular}

